\title{
Spontaneous Outflow Venous Thrombosis of an Unruptured Arteriovenous Malformation in the Setting of COVID-19 Infection
}

\author{
Camarano JG ${ }^{1}$, Hrushka JM ${ }^{1}$, Allison $R Z^{2}$, Robledo $A^{2}$, Raghuram $K^{3}$ and Kan $P^{2^{*}}$ \\ ${ }^{1}$ School of Medicine, University of Texas Medical Branch, USA \\ ${ }^{2}$ Department of Neurosurgery, University of Texas Medical Branch, USA \\ ${ }^{3}$ Department of Radiology, University of Texas Medical Branch, USA
}

*Corresponding author: Peter Kan, MD, Department of Neurosurgery, University of Texas Medical Branch, USA

\begin{abstract}
Background: First case reported of a partially thrombosed AVM in the setting of COVID-19 infection.

Observation(s): A 63-year-old man with a known left frontal AVM presented with a recent increase in seizure activity. Approximately two weeks prior to admission the patient was found seizing and febrile with a temperature of $38.2{ }^{\circ} \mathrm{C}$. At that time the patient had reported a one-week history of dry cough, shortness of breath and dyspnea on exertion. Nasopharyngeal swab testing with polymerase chain reaction (PCR) was positive for SARS-CoV-2. The patient's left frontal lobe AVM was first identified in 2007 with a nidus measuring approximately $21 \times 20 \times 21 \mathrm{~mm}$. Arterial supply was predominantly from small arterial branches arising from the pericallosal and callosomarginal branches of the left anterior cerebral artery. A large tortuous vein provided superficial drainage into the superior sagittal sinus.
\end{abstract}

On the current admission, computed tomography angiography (CTA) demonstrated an interval decrease in contrast enhancement within the venous varix. Magnetic resonance imaging (MRI) findings were also suggestive of partial thrombosis within this venous varix and demonstrated an interval increase in perivenous edema within the left superior frontal lobe. Four months later the patient underwent digital subtraction angiography (DSA), which revealed a significant decrease in nidus size, again suggesting partial thrombosis.

Here we report the first case of a patient with no known hypercoagulability disorder who presented with congestive cerebral edema and partial venous thrombosis of an unruptured cerebral AVM in the setting of COVID-19 infection.

\section{Keywords}

COVID-19, SARS-CoV-2, Arteriovenous malformation, Venous thrombosis, Hypercoagulable state, Infection

\section{Introduction}

Cerebral arteriovenous malformations (AVMs) are rare vascular anomalies with an estimated incidence of 1.3 per 100,000 persons/year [1]. The complex architecture of AVMs consists of arterial feeders which coalesce into a web of dysplastic vessels (nidus) which shunts blood flow directly into draining cerebral veins. The most common presentations are intracranial hemorrhage secondary to AVM rupture or seizures [2].

The degree of venous outflow is thought to contribute significantly to the hemodynamics within and surrounding the AVM nidus. Thus, stenosis or occlusion of draining veins are proposed as mechanisms predisposing to rupture $[3,4]$. Spontaneous thrombosis in AVM venous outflow is exceedingly rare but may occur secondary to compression or vasospasm following hemorrhage; turbulent flow due to aneurysmal dilation; or an underlying hypercoagulable state. Rarer is the presence of congestive peri-venous edema surrounding an unruptured $\mathrm{AVM}$, which may also occur secondary to venous obstruction.

Recent reports have indicated a high prevalence of complications from acquired coagulopathy in COVID-19 Infection. Neurosurg Cases Rev 4:088. doi.org/10.23937/2643-4474/1710088

Accepted: October 28, 2021; Published: October 30, 2021

Copyright: (c) 2021 Camarano JG, et al. This is an open-access article distributed under the terms of the Creative Commons Attribution License, which permits unrestricted use, distribution, and reproduction in any medium, provided the original author and source are credited. 
coronavirus disease 2019 (COVID-19) patients, including deep venous thrombosis (DVT), pulmonary embolism (PE), acute ischemic stroke, and cerebral vein thrombosis (CVT) [5]. Here we report the first case of a patient with no known hypercoagulability disorder who presented with congestive cerebral edema and partial venous thrombosis of an unruptured cerebral AVM in the setting of COVID-19 infection.

\section{Illustrative Cases}

\section{History \& presentation}

A 63-year-old man with a known left frontal AVM presented with a recent increase in seizure activity. Approximately two weeks prior to admission the patient was found seizing and febrile with a temperature of $38.2{ }^{\circ} \mathrm{C}$. At that time the patient had reported a oneweek history of dry cough, shortness of breath and dyspnea on exertion. Nasopharyngeal swab testing with polymerase chain reaction (PCR) was positive for SARSCoV-2.

On arrival, the patient was afebrile and hemodynamically stable. The only observed neurological abnormality was a mild resting tremor of the right hand and face. Laboratory abnormalities included an elevated D-dimer of 2,370 ng/mL (normal
$<500 \mathrm{ng} / \mathrm{mL}$ ), LDH of $993 \mathrm{U} / \mathrm{L}$ (normal 300-600 U/L) and CRP of $1.3 \mathrm{mg} / \mathrm{dL}$ (normal $<0.8 \mathrm{mg} / \mathrm{dL}$ ). A complete blood count, electrolyte levels and a coagulation panel were normal. Chest X-Ray revealed diffuse bilateral interstitial and airspace opacities in the mid and lower lung fields, findings typical of COVID-19 infection. Upon measurements of the patient's anti-epileptic medications, Levetiracetam was slightly sub-therapeutic (10 $\mathrm{\mu g} / \mathrm{mL}$; Normal therapeutic range: $20-40 \mathrm{mg} / \mathrm{L}$ ) while Phenytoin was within therapeutic range $(2.2 \mathrm{\mu g} /$ $\mathrm{mL}$; Normal therapeutic range: $1-2 \mathrm{mcg} / \mathrm{L}$ free drug).

\section{Current \& prior investigations}

The patient's left frontal lobe AVM was first identified in 2007 with a nidus measuring approximately $21 \times 20 \times 21 \mathrm{~mm}$. Arterial supply was predominantly from small arterial branches arising the pericallosal and callosomarginal branches of the left anterior cerebral artery. A large tortuous vein provided superficial drainage into the superior sagittal sinus. On follow up imaging, computed tomography angiography (CTA) demonstrated an interval decrease in contrast enhancement within the venous varix (Figure 1). Magnetic resonance imaging (MRI) findings were also suggestive of partial thrombosis within this venous varix and demonstrated an interval increase in vasogenic

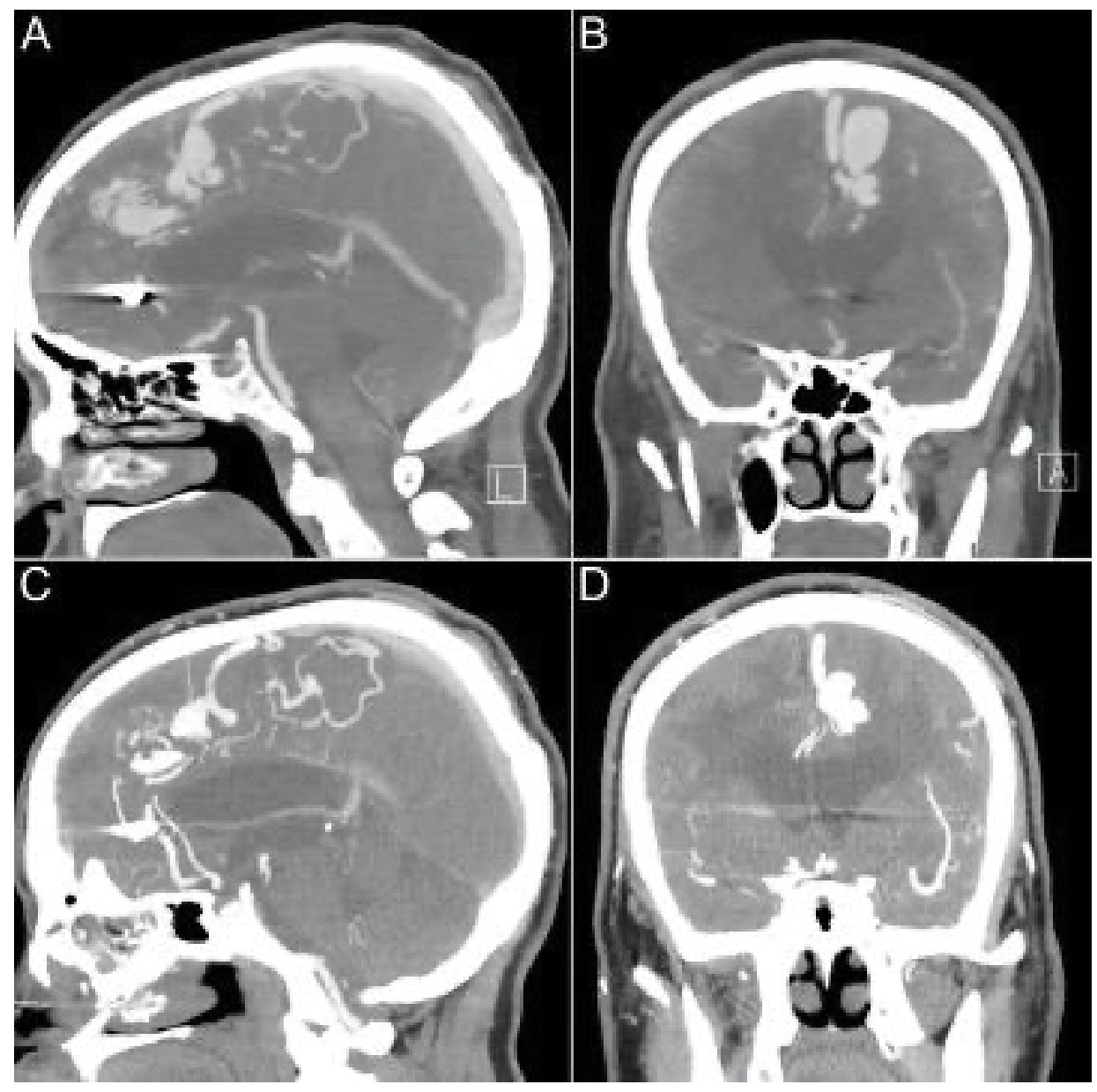

Figure 1: CT Angiogram in November of 2017 (Sagittal $[A]$, coronal [B]) and in October of 2020 (Sagittal [A], coronal [B]). The draining vein of the left frontal AVM is associated with a large saccular venous aneurysm. In $C$ and $D$, there is an interval decrease in contrast filling of the venous aneurysm. 

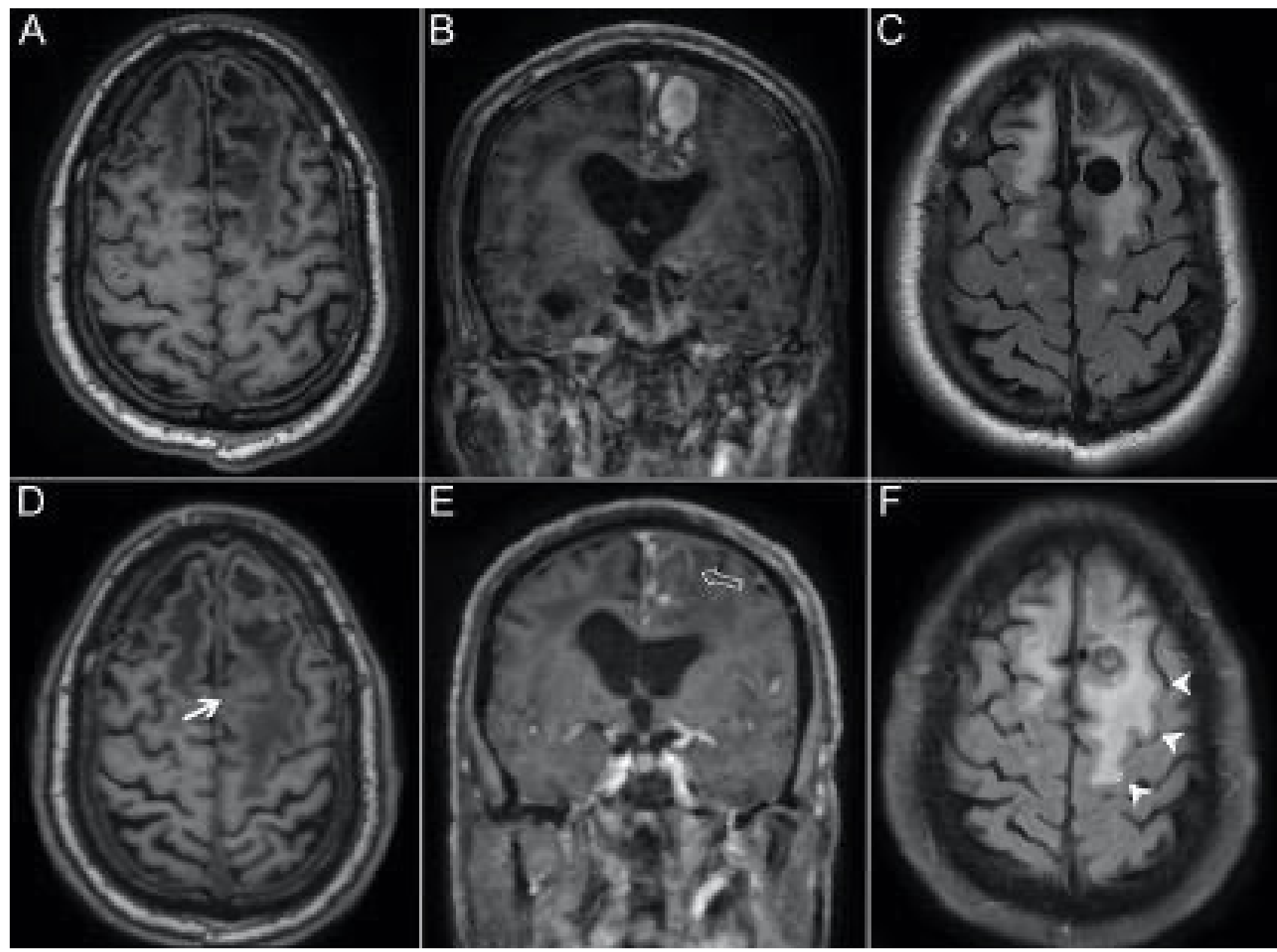

Figure 2: Axial T1 without gadolinium (A), Coronal T1 with gadolinium (B) and axial T2 of the brain undertaken in 2017 followed with axial T1 without gadolinium (D), coronal T1 with gadolinium (E) and axial T2 of the brain undertaken in October of 2020. (A \& D) Axial T1-weighted MRI imaging demonstrating an interval decrease in signal void within the draining vein aneurysm (bold arrow), suggesting partial thrombosis; (B \& E) Coronal T1-weighted MRI-imaging post-contrast demonstrating an interval loss of contrast enhancement within the venous aneurysm (arrow outline); (C \& F) Axial T2-FLAIR MRI demonstrating an interval increase in perivenous edema throughout the left superior frontal lobe (arrowheads).

perivenous edema within the left superior frontal lobe (Figure 2). Four months later the patient underwent digital subtraction angiography (DSA), which revealed a significant decrease in nidus size, suggesting partial thrombosis of the nidus in addition to draining vein thrombosis (Figure 3). Notably, the large ectatic cortical vein draining the residual nidus was now seen to empty into multiple convexity cortical veins, whereas it had previously drained predominantly into the superior sagittal sinus, again suggestive of interval thrombosis of the draining vein.

\section{Discussion}

Cerebral AVMs are high-flow, high-pressure tangles of dysplastic blood vessels through which arterial blood is shunted directly to venous drainage without a normal intervening capillary bed. Intracranial hemorrhage is the presentation in up to $58 \%$ of symptomatic patients, with each bleed associated with $10 \%$ mortality risk and $30-50 \%$ risk of neurologic defect $[6,7]$. As in our case, AVMs may present with seizures in up to $20-45 \%$ of patients [8-10].
Spontaneous occlusion (sometimes referred to as "spontaneous obliteration") of an AVM is uncommon, with estimated occurrence in only $1 \%$ of cases [11]. In most reported cases, it seems most likely that mass effect from hematoma or post-operative inflammation results in proximal compression of vessels near the nidus, producing turbulent blood flow that predisposes to thrombosis [12]. Additionally, vasospasm following subarachnoid hemorrhage may reduce blood flow within the AVM [11]. However, our patient showed no radiographic evidence of recent bleeding, thus excluding these mechanisms.

Spontaneous thrombosis of venous drainage in an unruptured AVM is a rare phenomenon; our literature review identified only 13 prior reports (Table 1 ). Of these, $77 \%$ were small lesions $(<3 \mathrm{~cm})$ and $69 \%$ demonstrated some form of venous outflow abnormality. Edema is another unique finding for these lesions and has been described in 3.3-3.9\% of unruptured AVMs $[13,14]$. Among the 13 reported cases of AVMs with venous thrombosis, 10 presented with some degree of edema, and all but one of these cases noted progression of 


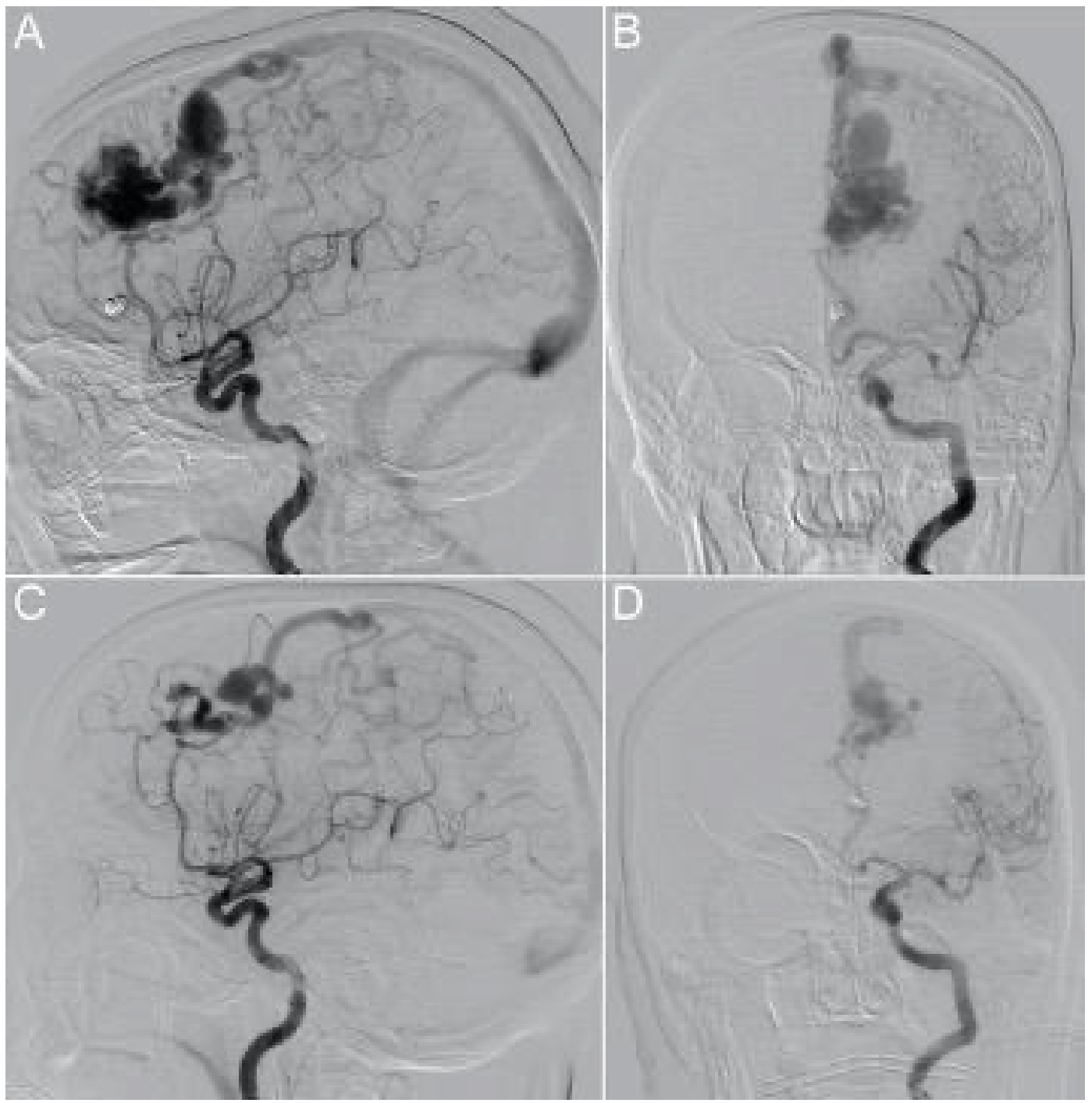

Figure 3: Left internal carotid artery injection in November of 2017 (lateral projection $[A]$, anterior-posterior projection [B]) and in October of 2020 (lateral projection [C], anterior-posterior projection [D]). The nidus is fed predominantly by a branch of the left pericallosal artery with minor contributions from the left callosomarginal artery. In A and B, drainage into the superior sagittal sinus is via a large tortuous vein with an associated venous pouch. $\ln C$ and $D$, there is significant reduction in the size of the AVM nidus. The residual nidus drains via a large posterior cortical vein which subsequently drains into multiple left cerebral convexity cortical veins. The draining vein's multiple ectatic segments have slightly changed in shape and anatomy compared to the previous study.

non-hemorrhagic symptoms. As such, the development of edema appears to be related to venous congestion secondary to thrombosis and stenosis of the draining vein. In such cases, proximal redistribution of blood to the weak plexiform vessels of the nidus may result in hemodynamic overload, contributing to the formation of edema and progression of non-hemorrhagic symptoms, which is believed to be the case in our patient [15-17]. A similar mechanism may contribute to an increased risk of hemorrhage $[3,18,19]$. However, several studies have suggested that the "silent" occlusion of draining veins is preceded by partial or complete obliteration of the lesion [11,12,20-22]. The anatomical and hemodynamic factors that may predispose an AVM to either rupture or regression following venous thrombosis remain poorly understood, but some authors suggest that AVMs that are small, superficial and have few arterial feeders may be more likely to spontaneously regress
[23]. Interestingly, the edema in our case predominantly expanded around the venous drainage tract rather than the nidus, a finding also reported by Kim, et al. [13]. This phenomenon cannot be explained solely by intranidal congestive hyperemia. In such instances, impaired drainage distal to the nidus may result in maladaptive changes to venous outflow in surrounding parenchyma, resulting in venous congestion within normal brain that shares the same drainage as the AVM [13].

In the absence of bleeding or venous stenosis, spontaneous thrombosis may occur in the presence of a hypercoagulable state. Although rare, cases have reported spontaneous venous thrombosis post-partum and in the setting of widespread cerebral venous thrombosis in a patient with an inherited Factor $V$ Leiden mutation [23-25]. In addition, heritable thrombophilia has been identified as a risk factor for cerebral venous (but not arterial) thrombosis [26]. This is the first 


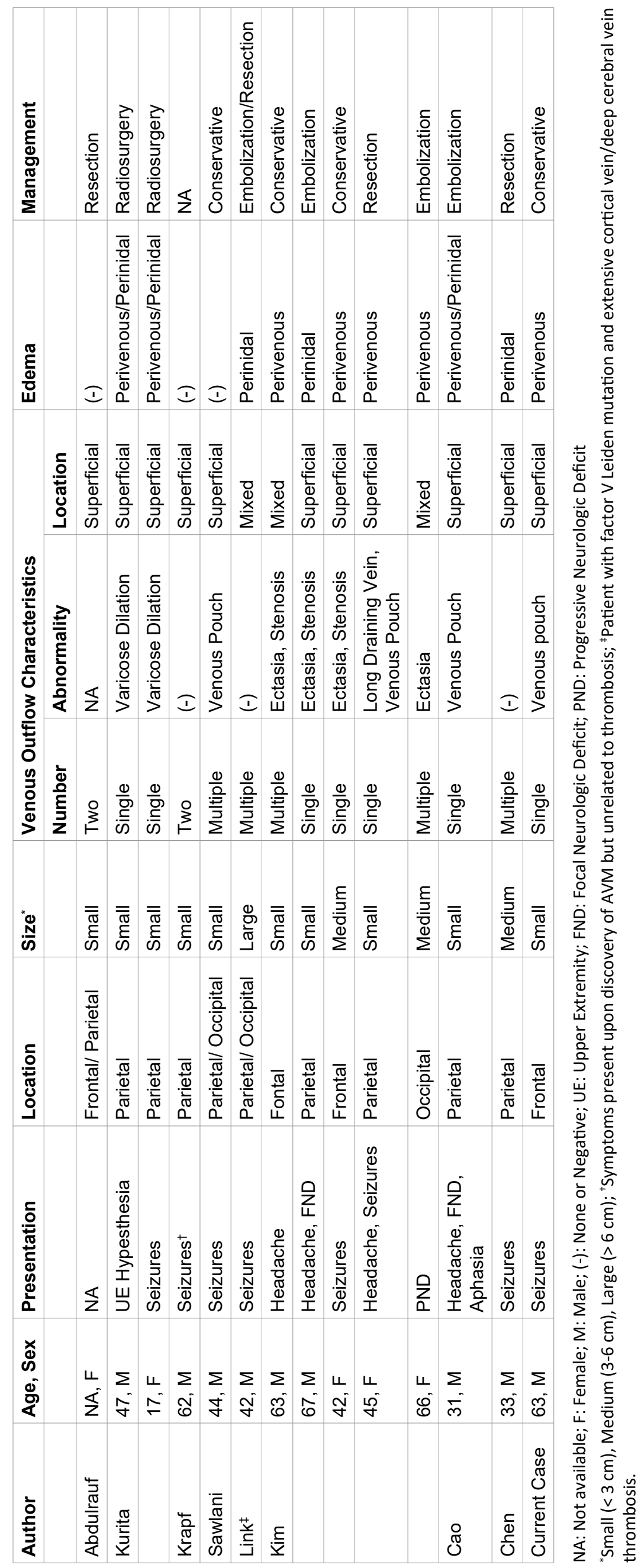


reported case of a partially thrombosed AVM in the setting of COVID-19 coagulopathy.

Although COVID-19 typically manifests with respiratory and systemic symptoms, venous and arterial thromboembolic complications have been widely reported among infected patients [5]. The hypercoagulable state induced by the virus is possibly due to hyperactivation of inflammatory factors in addition to viral tropism for angiotensin-converting enzyme 2 ( $A C E 2$ ) receptors present in the endothelium of blood vessels [27]. Laboratory abnormalities associated with COVID-19 coagulopathy include elevations in D-dimer, fibrinogen and LDH [28]. While these coagulation parameters have been found to be correlated to severity of the disease, they are also elevated in milder infection when compared to healthy controls [29]. Thrombocytopenia and abnormalities in PT/aPTT are commonly not seen [28]. Recently, several cases of both cerebral venous sinus and cortical vein thrombosis have been reported in COVID-19 patients, many of which presented with only mild respiratory or systemic symptoms [30-41]. As in our patient, the median time span between symptom onset and thrombotic event is 7 days [39].

In the current case, we believe venous thrombosis of the draining vein resulted in local edema secondary to venous stagnation and subsequent venous hypertension, with radiographic evidence of peri-venous edema coinciding with COVID-19 infection. This event corresponded with significant increase in edema and worsening of the patient's non-hemorrhagic symptoms. Although a causal relationship between infection and thrombosis cannot be definitively established, we suggest the infection to be a significant risk factor based on the temporal relationship of symptoms, laboratory evidence of COVID-19 hypercoagulability and plausible pathophysiologic mechanisms.

\section{References}

1. Al-Shahi R, Warlow C (2001) A systematic review of the frequency and prognosis of arteriovenous malformations of the brain in adults. Brain 124: 1900-1926.

2. Derdeyn CP, Zipfel GJ, Albuquerque FC, Cooke DL, Feldmann E, et al. (2017) Management of brain arteriovenous malformations: A scientific statement for healthcare professionals from the american heart association/American stroke association 48: e200-e224.

3. Hademenos GJ, Massoud TF (1996) Risk of intracrania arteriovenous malformation rupture due to venous drainage impairment. Stroke 27: 1072-1083.

4. Wilson CB, Hieshima G (1993) Occlusive hyperemia: A new way to think about an old problem. J Neurosurg 78 : 165-166.

5. Goswami J, Macarthur TA, Sridharan M, Pruthi RK, McBane $\mathrm{RD}$, et al. (2021) A review of pathophysiology, clinical features, and management options of covid-19 associated coagulopathy. Shock 55: 700-716.

6. Mast H, Young WL, Koennecke HC, Sciacca RR, Osipov
A, et al. (1997) Risk of spontaneous haemorrhage after diagnosis of cerebral arteriovenous malformation. Lancet 350: 1065-1068.

7. Martin NA, Vinters HV (1995) Arteriovenous malformations. In: Carter L, Spetzler R, Hamilton M. Neurovascular Surgery. McGraw-Hill, New York, 875-902.

8. Fennell VS, Martirosyan NL, Atwal GS, Kalani MYS, Ponce FA, et al. (2018) Hemodynamics associated with intracerebral arteriovenous malformations: The effects of treatment modalities. Neurosurgery 83: 611-621.

9. Lawton MT, Rutledge WC, Kim H, Stapf C, Whitehead KJ, et al. (2015) Brain arteriovenous malformations. Nat Rev Dis Prim 1: 15008.

10. Garcin B, Houdart E, Porcher R, Manchon E, Saint-Maurice JP, et al. (2012) Epileptic seizures at initial presentation in patients with brain arteriovenous malformation. Neurology 78: 626-631.

11. Abdulrauf SI, Malik GM, Awad IA (1999) Spontaneous angiographic obliteration of cerebral arteriovenous malformations. Neurosurgery 44: 280-287.

12. Hamada J, Yonekawa Y (1994) Spontaneous disappearance of a cerebral arteriovenous malformation: Case Report. Neurosurgery 34: 171-173.

13. Kim BS, Sarma D, Lee SK, Terbrugge KG (2009) Brain edema associated with unruptured brain arteriovenous malformations. Neuroradiology 51: 327-335.

14. Kumar AJ, Vinuela F, Fox AJ, Rosenbaum AE (1985) Unruptured intracranial arteriovenous malformations do cause mass effect. Am J Neuroradiol 6: 29-32.

15. Miyasaka Y, Yada K, Kurata A, Tokiwa K, Tanaka R, et al. (1994) An unruptured arteriovenous malformation with edema. Am J Neuroradiol 15: 385-388.

16. Shimizu S, Miyasaka Y, Tanaka R, Kurata A, Fujii K (1998) Pial arteriovenous malformation with massive perinidal edema. Neurol Res 20: 249-252.

17. Kurita H, Shin M, Ueki K, Kawamoto S, Kirino T (2001) Congestive brain oedema associated with a pial arteriovenous malformation with impaired venous drainage. Acta Neurochir (Wien) 143: 339-342.

18. Fukaya R, Mizutani K, Fukuchi M, Fujii K (2019) Thrombosis of the draining vein causes intracranial haemorrhage in the natural history of brain arteriovenous malformation: Case report. Br J Neurosurg 33: 94-95.

19. Field LR, Russell EJ (1995) Spontaneous hemorrhage from a cerebral venous malformation related to thrombosis of the central draining vein: Demonstration with angiography and serial MR. Am J Neuroradiol 16: 1885-1888.

20. Dyck P (1977) Spontaneous thrombosis of an arteriovenous malformation. Neurosurgery 287-290.

21. Megison P, Batjer HH, Purdy PD, Samson DS (1989) Spontaneous resolution of arteriovenous malformation without hemorrhage. AJNR Am J Neuroradiol 10: 204.

22. Mullan S (1994) Reflections upon the nature and management of intracranial and intraspinal vascular malformations and fistulae. J Neurosurg 80: 606-616.

23. Link MJ, Schermerhorn TC, Fulgham JR, Nichols DA (2004) Progressive neurological decline after partial spontaneous thrombosis of a Spetzler-Martin Grade 5 arteriovenous malformation in a patient with Leiden factor $\mathrm{V}$ mutation: Management and outcome. Case report. J Neurosurg 100: 940-945. 
24. Kontogiorgi M, Kalodimou V, Kollias S, Exarchos D, Nanas S, et al. (2012) Postpartum fatal cerebral vein thrombosis: A case report and review. Open J Clin Diagnostics 2: 1-3.

25. Oh MK, Ryu JH, Jeon WJ, Lee CW, Cho SY (2020) Case report: Postpartum cerebral venous thrombosis misdiagnosed as postdural puncture headache. BMC Anesthesiol 20: 80.

26. Walker ID, Greaves M, Preston FE (2001) Investigation and management of heritable thrombophilia. $\mathrm{Br} \mathrm{J}$ Haematol 114: $512-528$

27. Connors JM, Levy JH (2020) COVID-19 and its implications for thrombosis and anticoagulation. Blood 135: 2033-2040.

28. Ranucci M, Ballotta A, Di Dedda U, Baryshnikova E, Poli MD, et al. (2020) The procoagulant pattern of patients with COVID-19 acute respiratory distress syndrome. J Thromb Haemost 18: 1747-1751.

29. Han H, Yang L, Liu R, Liu F, Wu KL, et al. (2020) Prominent changes in blood coagulation of patients with SARS-CoV-2 infection. Clin Chem Lab Med 58: 1116-1120.

30. Klein DE, Libman R, Kirsch C, Arora R (2020) Cerebral venous thrombosis: A typical presentation of COVID-19 in the young. J Stroke Cerebrovasc Dis 29: 104989.

31. Hughes C, Nichols T, Pike M, Subbe C, Elghenzai S (2020) Cerebral venous sinus thrombosis as a presentation of COVID-19. Eur J case reports Intern Med 7: 001691.

32. Sugiyama $Y$, Tsuchiya $T$, Tanaka $R$, Ouchi A, Motoyama A, et al. (2020) Cerebral venous thrombosis in COVID-19associated coagulopathy: A case report. J Clin Neurosci 79: 30-32.

33. Hussain S, Vattoth S, Haroon KH, Muhammad A (2020) A case of coronavirus disease 2019 presenting with seizures secondary to cerebral venous sinus thrombosis. Case Rep Neurol 12: 260-265.
34. Hemasian H, Ansari B (2020) First case of Covid-19 presented with cerebral venous thrombosis: A rare and dreaded case. Rev Neurol (Paris) 176: 521-523.

35. Baudar C, Duprez T, Kassab A, Miller N, Rutgers MP (2020) COVID-19 as triggering co-factor for cortical cerebral venous thrombosis? J Neuroradiol 48: 65-67.

36. Roy-Gash F, Marine DM, Jean-Michel D, Herve V, Raphael B, et al. (2020) COVID-19-associated acute cerebral venous thrombosis: Clinical, CT, MRI and EEG features. Crit Care 24: 419.

37. Poillon G, Obadia M, Perrin M, Savatovsky J, Lecler A (2020) Cerebral venous thrombosis associated with COVID-19 infection: Causality or coincidence? J Neuroradiol 48: 121124.

38. Garaci F, Di Giuliano F, Picchi E, Da Ros V, Floris R (2020) Venous cerebral thrombosis in COVID-19 patient. J Neurol Sci 414: 116871.

39. Cavalcanti DD, Raz E, Shapiro M, Dehkharghani S, Yagh S, et al. (2020) Cerebral venous thrombosis associated with COVID-19. Am J Neuroradiol 41: 1370-1376.

40. Bolaji P, Kukoyi B, Ahmad N, Wharton C (2020) Extensive cerebral venous sinus thrombosis: A potential complication in a patient with COVID-19 disease. BMJ Case Rep 13: e236820.

41. Nwajei $F$, Anand $P$, Abdalkader M, Arasa VCA, Aparicio HJ, et al. (2020) Cerebral venous sinus thromboses in patients with sars-cov-2 infection: Three Cases and a Review of the Literature. J Stroke Cerebrovasc Dis 29: 105412. 